\title{
BioFibGel: A Green Nanotechnology Based Wound Dressing
}

\author{
Garima Shandilya ${ }^{1}$, Kirtan Tarwadi ${ }^{1}$, Sachin Chavan $^{1, *}$, Jai Singh Vaghela ${ }^{2}$ \\ ${ }^{1}$ Bharati Vidhyapeeth (Deemed to be University), College of Engineering, Pune, Maharashtra, INDIA. \\ ${ }^{2}$ Department of Pharmacy, Bhupal Nobles University, College of Pharmacy, Udaipur, Rajasthan, INDIA.
}

\begin{abstract}
Aim: Wound is a fragile and complex injury which can be as simple as a minor cut to the deep lesions and traumatic gash. Depending on the patient's internal and external conditions, wounds can progress backward or forward. Acute hemorrhage and wound infections are the main cause of loss of life in critical conditions of war field or in traumatic accidents. Innovation: BioFibGel bandage/dressing is a green nanotechnology based wound care product which is intended to be used in trauma, combat injuries (bleeding gunshot wounds) and mangling as first-line emergency tool. The dressing can absorb and retain significant amount of water without dissolving or losing their structure, stabilize blood flow and avert bleeding. Materials and Results: BioFibGel is silver nanoparticles based hydrogel sheets/ fibers made up of gelatin, glycerin and aloe vera which are considered as ideal materials for hemostasis activities and encourages cleaning and protection from reinjures or infection and speeds wound healing along with real-time monitoring of $\mathrm{pH}$ of wound using carbon dots fluorescence. Conclusion: This work is an attempt to devise a wound dressing that can aid in natural blood-clotting mechanism (sparing blood loss) and providing positive environment in wound healing.
\end{abstract}

Key words: Wound, Haemorrhage, Green nanotechnology, Silver nanoparticles, Wound dressing, Carbon dots.

\section{INTRODUCTION}

A wound dressing is aseptic covering provided to the wound to ensure fast and effective healing, reduce infection, scar or further harm. Dressings are intended to supply skin abrasion a sterile, breathable and moist conditions facilitating epithelialization and regeneration. These protective layers between wound bed and dressings forms an intermediate to acclimate optimum healing states throughout the way time of dressing. Diverse wound dressings are available in the markets which are differently applicable on different wounds. The right dressing could make a big difference in properly treating the wound.

Commercially available wound dressing materials such as hydrogels, films, nanoparticles incorporated dressings, hydrofiber, alginates and cellulose- hydrocolloids have been investigated and shown proven results in wound healing. Increasing interest for nanotechnology applications in medicine and wound care has revolutionized nontheoretical wound management.

Nanotechnology being means to overcome cellular or subcellular dimensions has acquired a special place in wound therapies by introducing innovative nanosystems and products in form of drug carriers, targeting agents, scaffolds as well as regeneration factors. BioFibGel is such an example of nanosystem in wound therapeutics. BioFibGel is a green nanotechnology based wound dressing with biologically synthesized silver nanoparticles subsumed in gelatin-based hydrofibers/ gel with aim to be used as haemostatic crises savior as well as dressing for wound management. This paper describes about BioFibGel concept, formulation and its performance for wound therapeutics.
Submission Date: 17-04-2021; Revision Date: 06-07-2021; Accepted Date: 24-08-2021

DOI: 10.5530/ijper.55.4.199 Correspondence:

\section{Dr. Sachin Chavan}

Bharati Vidhyapeeth Deemed to be University, College of Engineering,Pune-411043, Maharashtra, INDIA. Phone: +91-9271635407, Email: sschavan@bvucoep edu.in

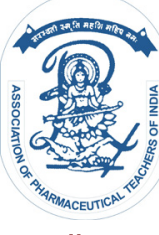

www.ijper.org 


\section{MATERIALS AND METHODS}

\section{Materials}

Silver nitrate $\left(\mathrm{AgNO}_{3}\right.$, bioreagent, solid) was purchased from Sigma-Aldrich, India. Gelatin from bovine skin (G9382, Type B, 225g bloom gel strength) purchased from Sigma-Aldrich, India. Glycerin (1.25 g/ml (lit.)) was purchased from Sigma-Aldrich, India. Curcuma longa was bought from the local grocery market, Rajasthan, India. Fresh aloe vera was used from home garden. Fresh lemon juice was used for biosynthesis of carbon dots from locally bought lemons.

\section{Methods}

Green biosynthesis of silver nanoparticles was done using Curcuma longa tuber powder or organic turmeric powder. Biogel was prepared separately using glycerin, gelatin, aloe-vera gel and deionized water. The experimental procedure is divided into three steps:

\section{Preparation of silver nanoparticles using organic turmeric powder}

Curcuma longa tuber was purchased from the local market, washed to remove any contamination and sun dried for seven days to remove all the water from the tuber. Tuber was grinded to get a fine powder and sieved using 20 micrometer mesh. To make turmeric extract $0.1 \mathrm{~g}$ of C. longa powder was taken added with $20 \mathrm{ml}$ deionized.

\section{Yellowish orange solution}

Water with vigorous stirring for $20 \mathrm{~min}$ at room temperature. 40 milliliters of $1 \mathrm{mM}$ silver nitrate then added to turmeric extract and mixed at room temperature till the colorless solution changes to dark brown solution, confirming formation of silver nanoparticles. ${ }^{1}$

\section{Biosynthesis of carbon dots using lemon juice}

Fresh lemon was cut and lemon juice was extracted from it. $40 \mathrm{ml}$ of filtered lemon juice was taken into glass crucible, covered with lid to prevent any loss and was microwaved for $6 \mathrm{~min}$ to complete the reaction. Dark brown-reddish mass was obtained confirming formation of carbon dots. ${ }^{2-3}$

\section{Extraction of Aloe Vera Gel}

Aloe vera was freshly cut from the plant, washed with distilled water to reduce contamination. Bark was removed with scalpel and gel sheets were obtained, subjected to mechanical stirring for $2 \mathrm{~min}$ and filtered to obtain juice. ${ }^{4}$

\section{Gel Matrix Preparation}

Gelatin, ${ }^{5}$ was purchased from the supermarket and medicinal glycerin was bought from a medical store.
Gelatin $(4 \mathrm{gm})$ was added to $100 \mathrm{ml}$ distilled water and was mixed for $1 \mathrm{hr}$ with continuous stirring at $80^{\circ} \mathrm{C}$. After that $40 \mathrm{ml}$ of freshly prepared aloe vera gel was mixed with continuous stirring for $30 \mathrm{~min}$. This gives us gel base. ${ }^{6,7}$ The process involved in gel matrix formation is sol-gel synthesis.

\section{Biofibgel synthesis}

The sol-gel product-gel base was then combined with other materials to deliver its characteristics. The Curcuma longa reduced silver nanoparticles (1\%) were added to the gel solution, ${ }^{8}$ and were mixed for $10-15 \mathrm{~min}$ till gel solution becomes slight greenish. This constitution is BioFibGel but to make it $\mathrm{pH}$ interactive and fluorescent, $1 \%$ carbon dots solution was mixed to the prepared solution and was stirred for 10-15 min till color changes to slight orange. Gel casting was done in petri dishes and was kept at $4^{\circ} \mathrm{C}$ until used. Figure 1 depicts the synthesis process of BioFibGel.

\section{RESULTS}

Reduction of silver to silver nanoparticles was visually observed by color change from yellowish orange to dark brown color in Figure 2 showing the synthesis of silver nanoparticles.. Gelatin ${ }^{9}$ was mixed well with aloe vera gel and glycerin to obtain a translucent solution named as gel base. Addition of silver nanoparticles to gel solution resulted in BioFibGel as the final product with slight color change to the gel matrix. Immersion of carbon dots to BioFibGel made them a $\mathrm{pH}$ interactive smart dressing with a tint of yellow color for the casted gel. Figure 3 depicts image showing petri dish casted BioFibGel samples.

\section{In-vitro analysis}

An ideal wound dressing exhibits special features to protect the wound and reconstruct physical hindrance

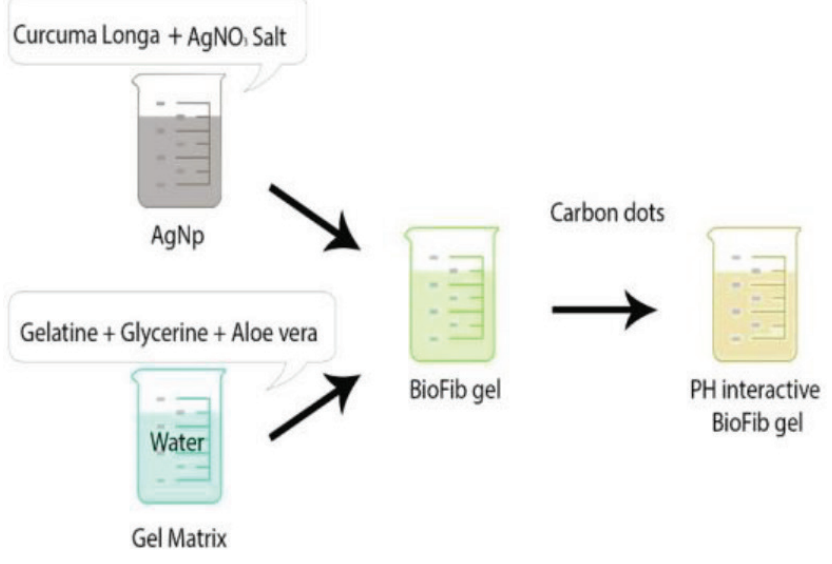

Figure 1: Synthesis process of BioFibGel. 


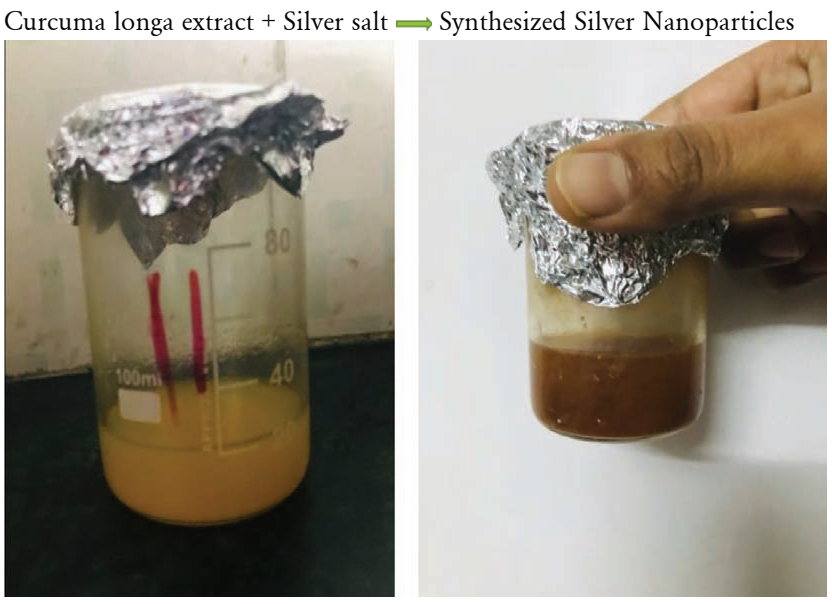

Figure 2: Synthesis of Silver Nanoparticles.

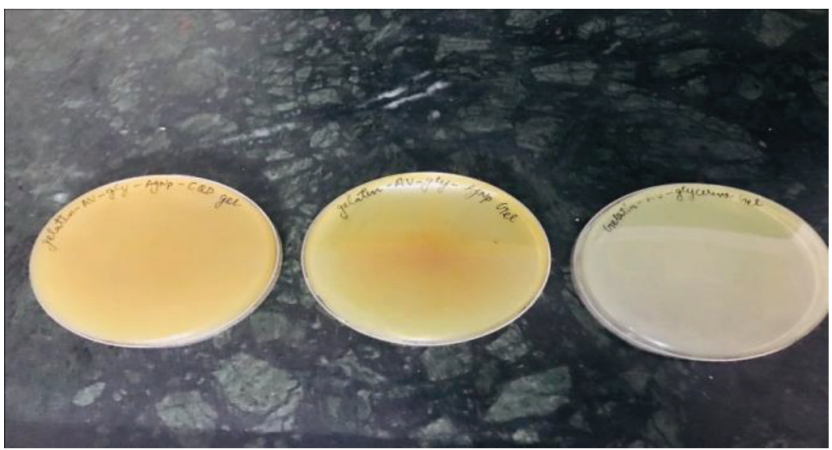

Figure 3: Image shows petri dish casted BioFibGel samples.

created by wound. There must be some properties of dressings like satisfactory mechanical strength to protect the wound from further damage, fluid uptake ability, healing agents transfer and a moist microenvironment to accelerate wound healing. Before any medical dressing is permitted to be used commercially it has to go through number of steps for in-vitro trials. The dressing material is investigated for various aspects of physical properties and for efficacy of dressings in wound management.

\section{Water-uptake capacity of wound dressing material}

This method is used to show how much capacity of fluid or exudate, a dressing could absorb to keep wound free from wound debriments while maintaining the $\mathrm{pH}$ of wound area. ${ }^{10}$ For this weight of wound dressing is taken before and after $24 \mathrm{hr}$ immersions in phosphate buffer solution (PBS) and using below formula water uptake capacity is calculated. ${ }^{11}$

$$
\text { Water Uptake } \%=\left[\left(\mathrm{W}_{\mathrm{o}}-\mathrm{W}_{1}\right) / \mathrm{W}_{\mathrm{o}}\right] \times 100
$$

$\mathrm{W}_{\mathrm{o}}=$ Weight of dry sample
$\mathrm{W}_{1}=$ Weight of sample after immersion in PBS at room temperature

Performing water uptake test on BioFibGel and $\mathrm{pH}$ interactive BioFibGel, it was found that carbon dots containing BioFibGel could hold upto $42.85 \%$ of water while BioFiGel could hold upto $71.42 \%$ of water. It was also observed that BioFibGel got flattened after soaking in water for so long whereas $\mathrm{pH}$ interactive BioFibGel maintained its shape throughout the test. The Figure 4 shows gels before and after soaking. It reveals the fact that carbon dots might have helped the gel matrix to maintain its shape even after soaking the water.

Porosity Measurement Porosity of wound dressing mats was analyzed using liquid displacement technique. Following equation is used. ${ }^{12}$

$$
\text { Porosity } \%=\left[\left(\mathrm{V}_{1}-\mathrm{V}_{3}\right) /\left(\mathrm{V}_{2}-\mathrm{V}_{3}\right)\right] \times 100
$$

Where,

$\mathrm{V}_{1}$ are initial volume of $96 \%$ ethanol

$\mathrm{V}_{2}$ is volume after scaffold was soaked in ethanol

$\mathrm{V}_{3}$ is volume of ethanol after scaffold removal

Thus, three weights, dry, wet and buoyant weight measures the porosity of dressing material. ${ }^{13,14}$ It was found that porosity of BioFibGel was $77.41 \%$ while $\mathrm{pH}$ interactive BioFibGel shows $62.50 \%$ porosity. This might be due to addition of carbon dots in $\mathrm{pH}$ interactive dressing that would have occupied the spaces in lattice structure of the gel matrix. In this test also BioFibGel was malleable by immersion in liquid while carbon dot incorporated

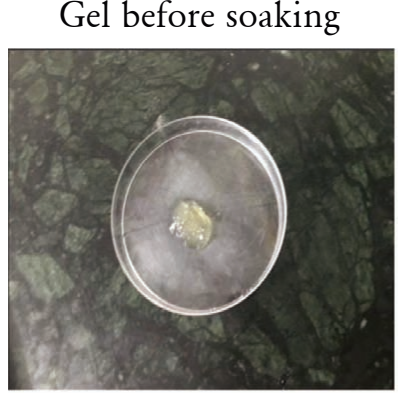

BioFibGel

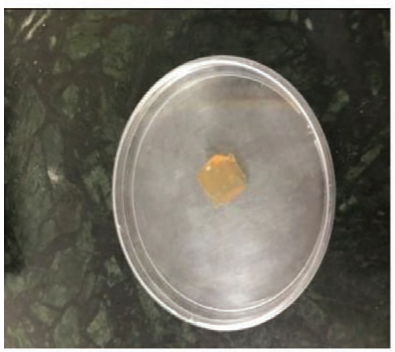

pH interactive BioFibGel

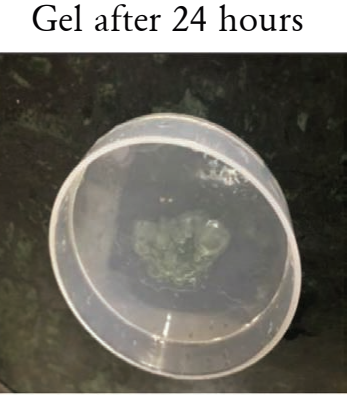

BioFibGel

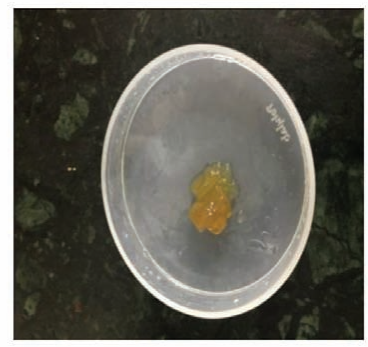

$\mathrm{pH}$ interactive BioFibGel
Figure 4: Gel water uptake capacity test. 

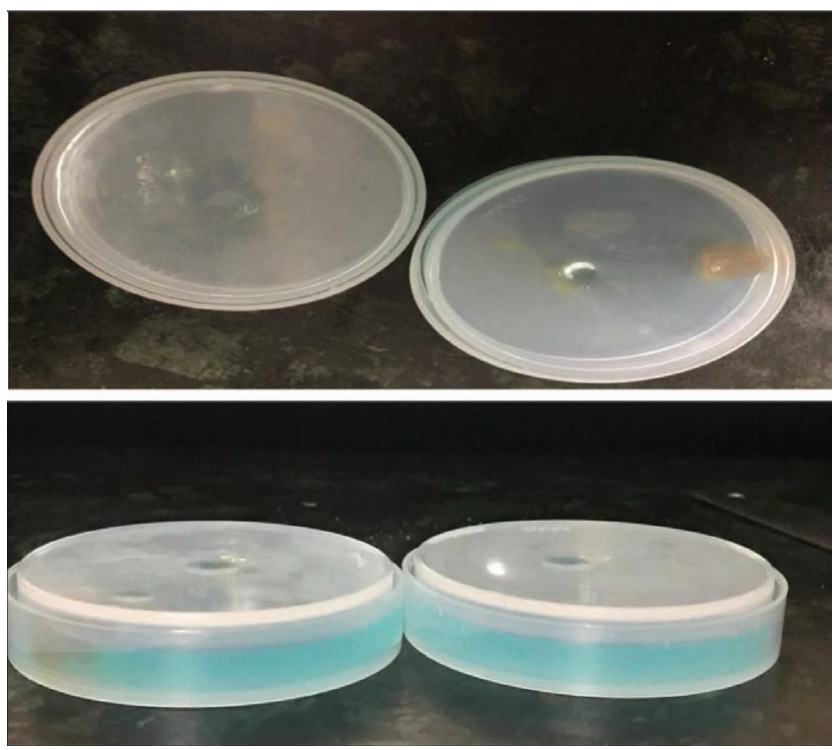

Figure 5: Samples under porosity test.

BioFibGel maintained its shape. The Figure 5 depicts the experimental glimpses for porosity measurement.

\section{Cell culture Analysis for Antibiotic action}

Antimicrobial activity of wound dressing material was determined using Wound PUS culture. The PUS sample from patients having wounds was collected by medical practitioner. Swabs were well soaked in pus and were taken in sterile zip-lock pouch to be transferred to lab for culturing. Frequency and number of sample collection was dependent on clinical cases of the patients with wounds. Pus swab was collected from all the types of wounds varying from accidental cases to recurrent ulcers to burn wounds. In laboratory agar petri dishes were used to perform PUS culture on wound swabs.

Kirby-bauer method, ${ }^{15}$ was used for analyzing antimicrobial efficiency of wound dressing formulations. PUS swab was distributed on 96 well agar plates with different sections for samples to be tested and was then incubated for $72 \mathrm{hr}$ at $37^{\circ} \mathrm{C}$. Table 1 shows the five samples which were tested for their antimicrobial action towards wound pus.

It was founded from Kirby-bauer results that BioFibGel and $\mathrm{pH}$ interactive BioFibGel shows a good antimicrobial action towards PUS swab culture of different wounds. It can be stated that dressings with antimicrobial agents are more effective towards resisting bacterial load. The $\mathrm{pH}$ interactive BioFibGel has shown bacterial resistivity up to an area of $3 \mathrm{~mm}-7 \mathrm{~mm}$ for differential samples. Some of the images of pus culture results are mentioned below in Figure 6. This culture assay is based on diffusion of antimicrobial agent from sample
Table 1: Samples and their formulations used to perform wound PUS culture.

\begin{tabular}{|c|c|}
\hline Sample & Formulations \\
\hline Control & Gelatin+Aloe Vera+Glycerin \\
\hline BioFibGel & Gelatin+Aloe Vera+Glycerin+Ag Np \\
\hline $\mathrm{pH}$ interactive & Gelatin+Aloe Vera+Glycerin+Ag \\
BioFibGel & Np+Carbon dots \\
\hline Ointment & Nanocrystalline silver nanoparticle \\
& Feracrylum \\
\hline
\end{tabular}
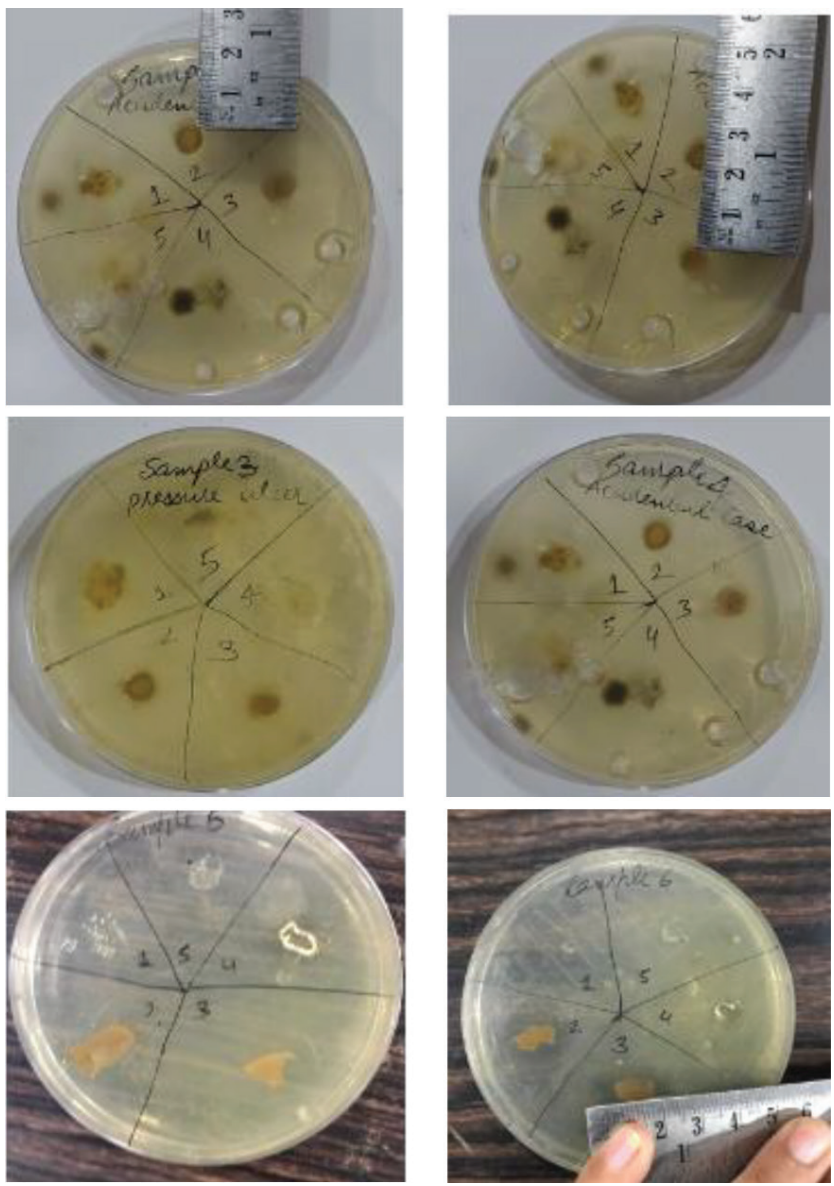

Figure 6: Samples with Kirby test results.

into agar gel. The sensitivity of sample was measured in strain of size of inhibition zone around the placed sample. It was found that microbes which were more resistant to antimicrobial action of sample were grown at shorter distance from the sample placed while less resistant microbes grew at greater distant from sample placed.

\section{In-vitro pH monitoring using carbon dots}

Carbon dots were synthesized through microwave assisted hydrothermal treatment of lemon juice. Different 


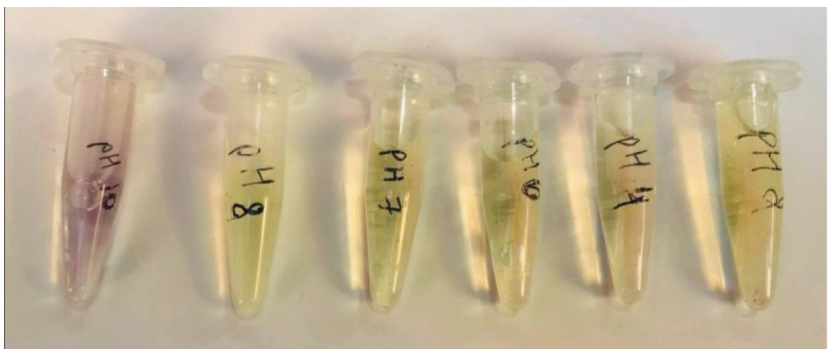

Carbon dots in different $\mathrm{pH}$ solutions under UV light

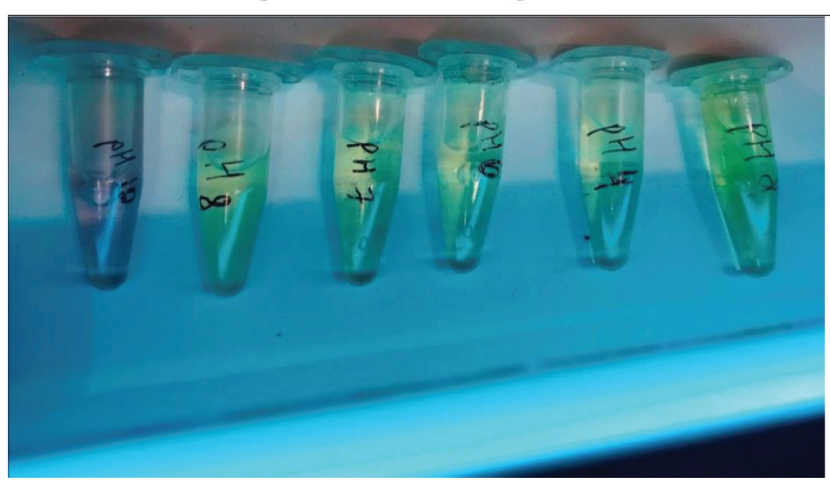

Figure 7: Carbon dots in different pH in daylight and under UV.

$\mathrm{pH}$ value solution was prepared to test the fluorescence of carbon quantum dots under UV (Ultra violet) light. The solutions of $\mathrm{pH}$ value $2,4,6,8$ and 10 were prepared using $\mathrm{HCl}$ for acidic medium and $\mathrm{NaOH}$ for basic medium by serial dilution. Carbon quantum dots were then added to the solutions and these solutions were observed under UVA $(320-400 \mathrm{~nm})$ to monitor fluorescence at different $\mathrm{pH}$. Figure 7 shows the image of different solution in daylight and under UV.

\section{DISCUSSION}

BioFibGel has unique gelling property, locking in exudate and its harmful component, contouring wound surface and responding to wound environment by forming a cohesive gel. Some of the properties are mentioned as:

- Locks in: BioFibGel when comes in contact with wound, dressing swells by absorbing bleeding and exudate, locking in harmful components, bacteria and enzymes, protecting peri-wound skin and reducing risk of maturation.

- Contour: BioFibGel forms a soft cohesive gel which contours over surface of wound providing a protective environment for wound healing. This indicates that all the spaces between wound bed and dressing are completely filled, leaving no area for bacteria to proliferate as well as protecting nerve endings from drying out.
- Response: BioFibGel when applied to wound bed responds by gelling the dressing and forming a cohesive gel that will maintain the moisture balance, flow of exudate from wound to dressing, transfer of healing agents and loaded drugs from dressing to wound site, maintaining optimum temperature and $\mathrm{pH}$ throughout the way time of dressing.

- Silver nanoparticles: Addition of silver nanoparticles shows a broad spectrum of anti-microbial and anti-fungal activity by fighting against bacteria or enzyme prevailing in dressing due to exudate absorption.

- Carbon quantum dots: Introduction of carbon quantum dots serves two purposes in the BioFibGel. First, it enhances the anti-microbial activity of the dressing in amalgamation with silver nanoparticles. Secondly, carbon quantum dots are highly luminescent; therefore they are used to show colorimetric response to monitor $\mathrm{pH}$ change of the wound. Sensitivity of fluorescent carbon dots is capable to show $\mathrm{pH}$ range of wound, biocompatibility and drug compatibility are remarkable properties to incorporate in a wound dressing.

- Easy application and removal of dressing: Being a gel pad, BioFibGel can be easily applied on the wound surface and after performing its task, it results in smooth and painless removal of dressing.

\section{CONCLUSION}

BioFibGel is a green nanotechnology wound dressing made from biosynthesis of silver nanoparticles and green method to make the gel matrix from gelatin. Gelatin is a unique combination of amino acids derived from collagen. As collagen is abundant in skin, bones, tendons, ligaments, providing strength and structure to tissues, gelatin thus helps in creating new tissues and skin as wound heals. Addition of aloe-vera has enlarged the pore size of the matrix with increased porosity to aid in exchange of fluids between wound bed and gel matrix. Glycerin is a natural humectant which exhibits desirable properties which contribute to suitable wound healing environment. Silver nanoparticles were loaded to gel matrix as anti-microbial and anti-fungal agents thus protecting wound from attacks of microbes. BioFibGel when applied to wound has aided to provide good anti-microbial action, positive healing environment and ensures rapid wound recovery with smartly monitoring $\mathrm{pH}$ of wound through carbon dot fluorescence mechanism. 


\section{ACKNOWLEDGEMENT}

We want to thank Bharati Vidhypeeth Deemed to be University and Bhupal Nobles College of Pharmacy for providing us with steadfast support throughout this study.

\section{CONFLICT OF INTEREST}

The authors declare no conflict of interest.

\section{ABBREVIATIONS}

UV: Ultra Violet rays; $\mathbf{H C l}$ : Hydrochloric acid; $\mathbf{N a O H}$ : Sodium Hydroxide; pH: Power of Hydrogen; PBS: Phosphate Buffer Solution; AgNO$_{3}$ : Silver Nitrate; AgNp: Silver Nanoparticles; mM: Millimolar; ml: Milliliter; g: Gram.

\section{REFERENCES}

1. Shameli Kamyar, Ahmad Mansor Bin, Zamanian Ali, Sangpour Parvanh, Shabanzadeh Parvaneh, Abdollahi Yadollah, Zargar Mohsen. Green biosynthesis of silver nanoparticles using Curcuma longa tuber powder. Int J Nanomedicine, (). 2012;7:5603-10- doi:10.2147/IJN.S36786, doi: 10.2147/ IJN.S36786, PMID 23341739.

2. Hoan BT, Tam PD, Pham V-H. Green synthesis of highly luminescent carbon quantum dots from lemon juice. J Nanotechnol. 2019;2019:1-9. doi: 10.1155/2019/2852816.

3. Monte-Filho Severino S, Andrade Stefani IE, Lima Marcelo B, Araujo Mario CU. Synthesis of highly fluorescent carbon dots from lemon and onion juices for determination of riboflavin in multivitamin/mineral supplements. J Pharm Anal. 2019;9(3):209-16. doi: 10.1016/j.jpha.2019.02.003, PMID 31297299.
4. Make aloe vera gel. Available from: https://www.wikihow.com/Make-AloeVera-Gel [cited 2/8/2021].

5. Gelatin. Available from: https://www.healthline.com/nutrition/gelatin-benefits \#section1 [cited 2/8/2021].

6. Hydrogel wound dressing. Available from: https://www.bandagesplus.com/ how-to-use-hydrogel-dressings [cited 2/8/2021].

7. Stout Edward I, McKessor Angie. Glycerin-based hydrogel for infection control. Adv Wound Care. 2012;1(1):48-51. doi: 10.1089/wound.2011.0288. PMID 24527279.

8. Rai Mahendra, Yadav Alka, Gade Aniket. Silver nanoparticles as a new generation of antimicrobials. Biotechnol Adv. 2009;27(1):76-83. doi: 10.1016/j. biotechadv.2008.09.002, PMID 18854209

9. Blanco-Fernandez Barbara, Castaño Oscar, Mateos-Timoneda Miguel Ángel, Engel Elisabeth, Pérez-Amodio Soledad. Nanotechnology approaches in chronic wound healing. Adv Wound Care (New Rochelle). 2021;10(5):23456. doi: 10.1089/wound.2019.1094, PMID 32320364.

10. Arslan Aysu, Simşek Murat, Aldemir Sevcan Dalkıranoğlu, Kazaroğlu Nur Merve, Gümüşderelioğlu Menemşe. Honey-based PET or PET/chitosan fibrous wound dressings: effect of honey on electrospinning process. J Biomater Sci Polym Ed. 2014;25(10):999-1012. doi: 10.1080/09205063.2014.918455, PMID 24842308.

11. Wienk IM, Folkers B, van den Boomgaard T, Smolders CA. Critical Factors in the Determination of the Pore Size Distribution of Ultrafiltration Membrances Using the Liquid Displacement Method. Sep Sci Technol. 1994;29(11):1433-40. doi: 10.1080/01496399408003029.

12. Samadian Hadi, Salehi Majid, Farzamfar Saeed, Vaez Ahmad, Ehterami Arian, Sahrapeyma Hamed, Goodarzi Arash, Ghorbani Sadegh. In vitro and in vivo evaluation of electrospuncellulose acetate/gelatin/hydroxyapatitenanocomposite mats for wound dressing applications. Artif Cells Nanomed Biotechnol. 2018;46(sup1);Suppl 1:964-74. doi: 10.1080/21691401.2018.1439842, PMID 29458271.

13. Dane, J. H., Topp, C. G., Flint, L. E., \& Flint, A. L. (2002). 2.3 Porosity. SSSA Book Series. doi:10.2136/sssabookser5.4.c11

14. Formula of porosity? - research Gate.

15. Kirby bauer method- http://shs-manual.ucsc.edu/policy/kirby-bauer-antibioticsensitivity\#: :text=The\%2OKirby\%2DBauer\%20test\%2C\%20known,growth\%20 measured\%20under\%20standard\%20conditions.

\section{SUMMARY}

- BioFibGel is found to be an interactive wound dressing which has done remarkable upgradation in current wound care products. The natural, non-toxic and biocompatible material, gelatin as external layer protection has performed better than collagen/hydrogels in healing cuts as well as burns. BioFibGel was found better in porosity and water uptake capacity but could not be able to maintain its shape after liquid interaction while carbon dots incorporated BioFibGel have appreciable amount of porosity including water uptake capacity sufficient for our application in absorbing exudate while maintaining its shape.

- Two different agents silver nanoparticles and carbon dots added to gelatin matrix were observed from Kirby Bauer method for antimicrobial action. It was observed that combination of both in $\mathrm{pH}$ interactive BioFibGel yielded effective antimicrobial action than other counterparts in keeping durable sterile surroundings. Superficial $\mathrm{pH}$ monitoring of wound with carbon dots fluorescence characteristics was confirmed by viewing different $\mathrm{pH}$ solutions under UV.

- As a result, we could say that the natural biocomposite dressing- BioFibGel has checklisted its entire objectives to be a potent tool in wound management. 


\section{PICTORIAL ABSTRACT}

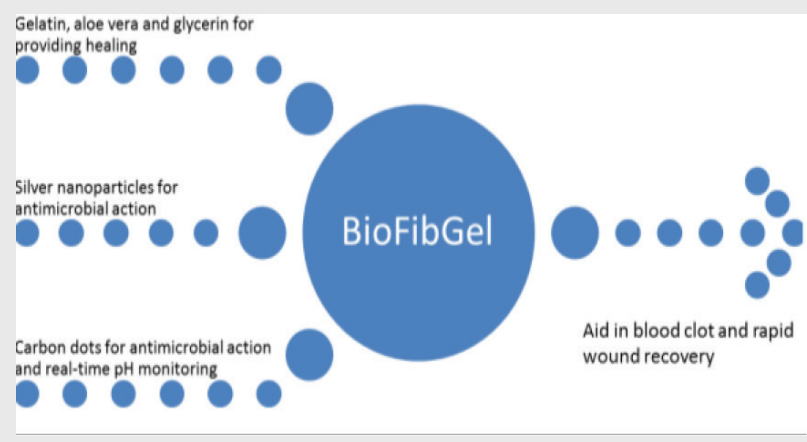

\section{About Authors}

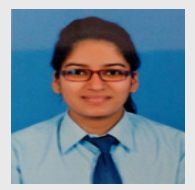

Garima Shandilya: M.Tech Scholar, Bharati Vidhyapeeth (Deemed to be University), College of Engineering, Pune, Maharashtra, India. She is working in the field of Nanotechnology and its applications in nanomedicine, nanoagronomics and nanobiotechnology.

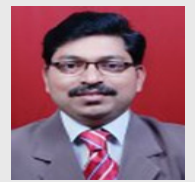

Sachin Chavan: Professor, Bharati Vidhyapeeth (Deemed to be University), College of Engineering, Pune, Maharashtra, India. His research area of interest is nanotechology, Nano fibers synthesis and their various applications.

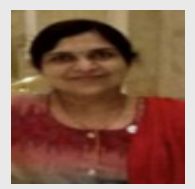

Kirtan Tarwadi: Professor, Bharati Vidhyapeeth (Deemed to be University), College of Engineering, Pune, Maharashtra, India. Her research areas are biotechnology, biochemistry and nanotechnology.

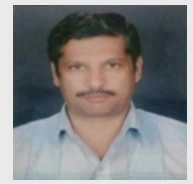

Jai Singh Vaghela: Associate Professor, Bhupal Noble College of Pharmacy, Udaipur, Rajasthan, India. His area of specialization is Pharmacology, Antiulcer, hepatoprotective, antioxidant and immune modulatory activities.

Cite this article: Shandilya G, Tarwadi K, Chavan S, Vaghela JS. A Green Nanotechnology Based Wound Dressing. Indian J of Pharmaceutical Education and Research. 2021;55(4):989-95. 